Pain Physician. 2004;7:133-139, ISSN 1533-3159

\title{
ImAgING In LUmbar SPInAL StenOSIS
}

Joseph D. Fortin, DO, and Michael T. Wheeler, DO

Lumbar spinal stenosis is a common condition seen in patients presenting to physicians who specialize in pain management or perform spine surgery. The designation of "spinal stenosis" without other qualifiers is vague and as such holds little practical value. Classifications have been created in order to more specifically describe the various etiol- ogies as well as the site(s) of narrowing. For this purpose, diagnostic imaging studies are vital. These include myelography, computed tomography (CT), and magnetic resonance imaging (MRI). Each imaging modality has its own inherent advantages and limitations in demonstrating anatomical structures and how they may contribute to the stenotic process.
Since proper treatment follows accurate identification of the pathology, it is important for physicians to have a sound understanding of normal and abnormal spinal elements as they are depicted on various imaging studies.

Keywords: Lumbar spinal stenosis, computed tomography, magnetic resonance imaging, myelography
Spinal stenosis is the focal, segmental, or diffuse narrowing of the central canal or root canals by bony and/or soft tissue elements, resulting in encroachment on neural structures(1). The goal of imaging studies in evaluating patients with symptoms potentially resulting from spinal stenosis is two-fold: 1) to define relative contributions of each anatomic component to the stenotic process, and 2) to help relate structural alterations to the change in the patient's ability to function. To that end, technological advances have allowed clinicians to view spinal anatomy with ever increasing resolution (Fig. 1). The future promises more functional applications such as devices which reveal dynamic disk herniations and open systems affording the dynamic evaluation of the spine in various positions. While technological advancements have enhanced and diversified the role of imaging studies, their basic focus should be a diagnostic extension of the clinical context. Denying the limitations can lead to disaster - for example, solely directing one's focus toward a compelling (yet asymptomatic) disc herniation when the underlying cause of the patient's back pain is an aortic

From Spine Technology and Rehabilitation, PC, Fort Wayne, IN. Address Correspondence: Joseph D. Fortin, DO, Spine Technology and Rehabilitation, 7230 Engle Road, Suite \#210, Fort Wayne, IN 46804.

E-mail: fortin@pol.net

Funding: There was no outside financial support for this project.

Conflict of Interest: None aneurysm, lymphoma, or prostate cancer.

\section{Defining Spinal Stenosis}

The word stenosis has its origin from the Greek stegnosis, which indicated complete obstruction of ducts or orifices of the body. Eventually, it was used to describe the impeded passage of iodized oil during myelography. The term stuck despite later attempts by the same author to change it to "narrowing," which he felt to be more representative of a process involving the walls of a container and the effect on its contents rather than obstruction by the contents itself (1). Currently, spinal stenosis is applied to describe narrowing of any spinal neuroaxial canal caused by one or several possible anatomic elements in association with a number of possible etiologies (2). It is therefore a condition rather than a disease in the strictest sense. The compression created by such narrowing, however, may indeed cause disease, as in myelopathy and radiculopathy.

The spinal neuroaxial canals include the central canal, lateral recess or entry zone, intervertebral foramen or mid-zone, and exit zone $(3,4)$. Techniques used to quantify central stenosis and determine the extent considered significant have been investigated and debated. Utilizing roentgenograms, the earliest techniques measured the interpedicular distance on the anteroposterior (AP) projection and central canal width on the lateral projection. A set of normative values was generated for reference. The measurements were subject to error secondary to improper patient positioning during film acquisition (magnification effect). The Torg ratio was later devised to obviate the technical precision required for accurate measurement. It represents the central canal width divided by the width of the anterior column. Since it is a relative measurement, magnification effect is negated. However, standard radiographic evaluation of spinal stenosis remained limited by measurement inaccuracies (e.g., overlying bony densities) and the lack of soft tissue appreciation. Consequently, myelography became the gold standard for the delineation of spinal stenosis. With the emergence of CT and MRI, greater anatomical detail visualized in multiple planes provided a better means for accurately measuring central stenosis. Currently, the most widely accepted technique is the measurement of the midline osseous sagittal (AP) diameter as described by Verbiest. Less than $12 \mathrm{~mm}$ is considered relative stenosis, and less than $10 \mathrm{~mm}$ is considered absolute stenosis. This is optimally obtained from a mid-pedicle axial section orthogonal to the long axis of the central canal $(1,5)$. For this purpose, either MRI or CT may be used as both have their advantages and disadvantages. Utilizing sagittal views may lead to measurement error since determination of true midline is more difficult. Lateral recess and foraminal stenoses are not as well quantified and are still evaluated subjectively (mild, moderate, or severe). 


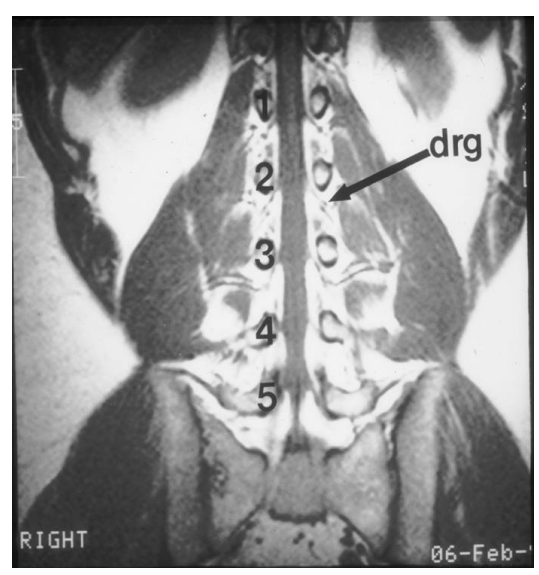

Fig 1. The T1-weighted lumbar MRI coronal view of this patient accurately depicts the normal anatomy of the segmental nerve roots as they depart the thecal sac adjacent the pedicle (entry zone or lateral recess), course under the pedicle (mid-zone, wherein lies the dorsal root ganglion), and travel beyond the pedicle (exit zone, which houses the ventral ramus)

\section{Classification of Stenosis}

Spinal stenosis can be classified as congenital, developmental, acquired, or combined $(1,5)$. Congenital stenosis is due to disturbed fetal development resulting in malformation of the lumbar spine. Examples include myelodysplasia, diastematomyelia, and hemivertebrae (Fig. 2). Developmental stenosis is a growth disturbance of the posterior elements involving the pedicles, lamina, and/or articular processes which results in decreased volume afforded to the spinal cord (or cauda equina in the case of the lumbar spine).
Examples include malrotation of the posterior elements, achondroplasia, and congenitally short pedicles (Fig. 3). Acquired stenosis denotes changes that occur after skeletal maturity that produce narrowing in a heretofore-normal spine. Causes can be separated into four groups: 1) traumatic, 2) degenerative, 3) infectious, and 4) neoplastic. Spondylolisthesis due to fracture of the pars interarticularis is the prototypical trauma-induced stenosis, but post-surgical and post-traumatic hypertrophic changes are also implicated. Discopathy and spondylosis represent degenerative processes associated with aging (6), as well as those accelerated by disease states (e.g., Paget's disease) (1). By far, they constitute the majority of acquired stenosis cases. Conversely, infectious and neoplastic etiologies are rare but serious cases that must be ruled out if the clinical presentation is suggestive. Combined stenosis is, quite simply, the result of acquired changes (usually degenerative) superimposed on a congenitally or developmentally narrow canal (Fig. 4). Normally, the reserve capacity of the spinal canal is sufficient to accommodate encroachment from adjacent structures, given that this is a relatively slow and limited process. In the context of combined stenosis, the reserve capacity is compromised such that relatively minor pathological processes may produce dramatic symptoms (Fig. 5). Some authors stipulate that all cases of symptomatic "acquired" stenosis (secondary to degenerative processes) are more appropriately designated as combined stenosis, with some degree of precursory narrowing necessary to become manifest (1).

\section{Anatomical Contributions}

\section{Anterior Column}

Structures of the anterior column may be implicated in the development of spinal stenosis via architectural derangement, mass affect, or both. Degenerative disc disease and loss of the normal intervertebral height will consequently reduce the vertical dimension of the root canals (i.e., foraminal stenosis). Additionally, buckling of the posterior longitudinal ligament may cause central stenosis (3). Mass affect is usually equated with disc herniation by lay people and many physicians. While this is certainly contributory, the space-occupying affect of degenerative osseous formations plays a significant and under appreciated role, especially in patients over forty years old (7). Osseous formations of the anterior column typically present as ridges and spurs stemming from the vertebral endplates. They may be indicative of compensatory load redistribution in the failing motion segment(s) according to Wolff's law (Fig. 6). Of course, any traumatic or infiltrative process causing expansion of the osseous cortical margins may also compromise canal dimensions. Ossification of the posterior longitudinal ligament (OPLL) is a distinct and uncommon etiology of bony central stenosis. Both discopathy and spondylosis can cause foraminal and/or central stenosis, depending on the site of the pathology (Fig. 7), and often in combination with one another. Spondylosis results only in "hard" stenosis that is relatively static and not subject to deformation. Discopathy may produce either "soft" or "hard" stenosis depending
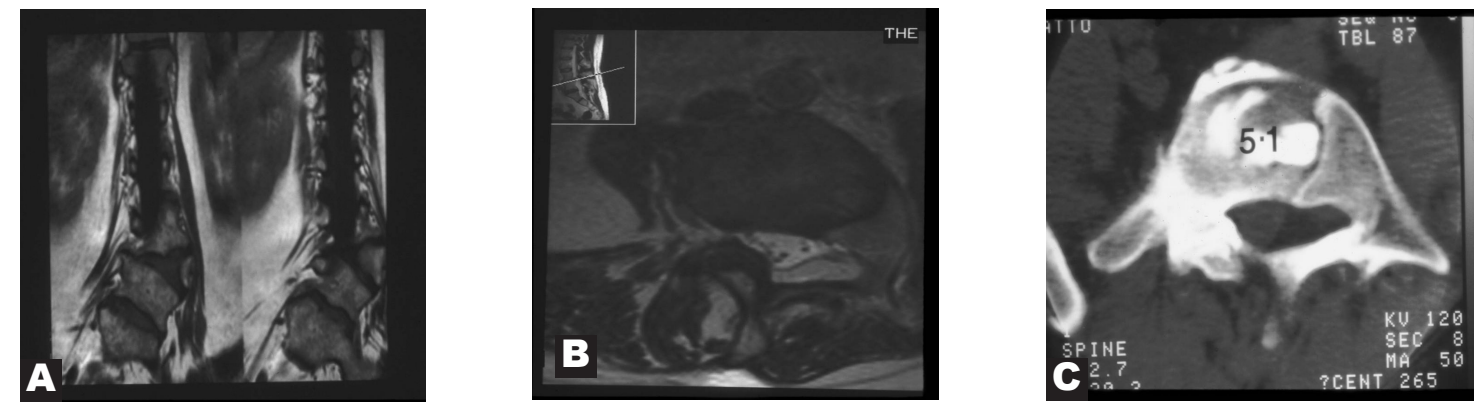

Fig 2. The hemivertebrae anomaly distorts the central canal configuration. (A)Coronal T1-weighted MRI pulse sequence. (B) T2-weighted axial pulse sequence. (C) Post-discography CT axial section through the L5-S1 disc (bone window settings) 


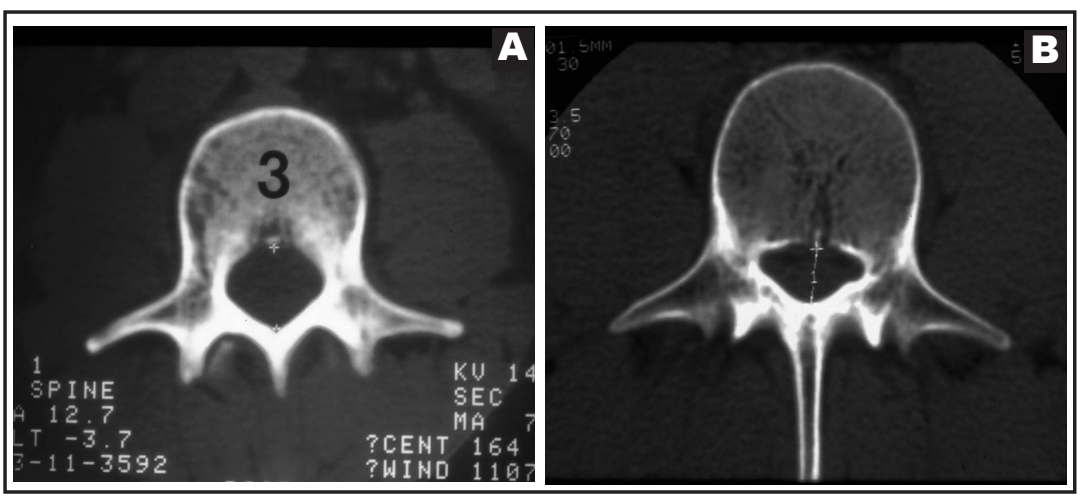

Fig 3. These T1-weighted axial images were acquired before (A) and after (B) application of a Dyanawell compression device. This patient with congenitally short pedicles and L2-3 posterior annular prominence (as evidenced by the convex versus the usual concave contour of the posterior annulus) is predisposed to neural compression with weight bearing activities. Note the change in the volume of the central canal (the white encircled thecal sac)

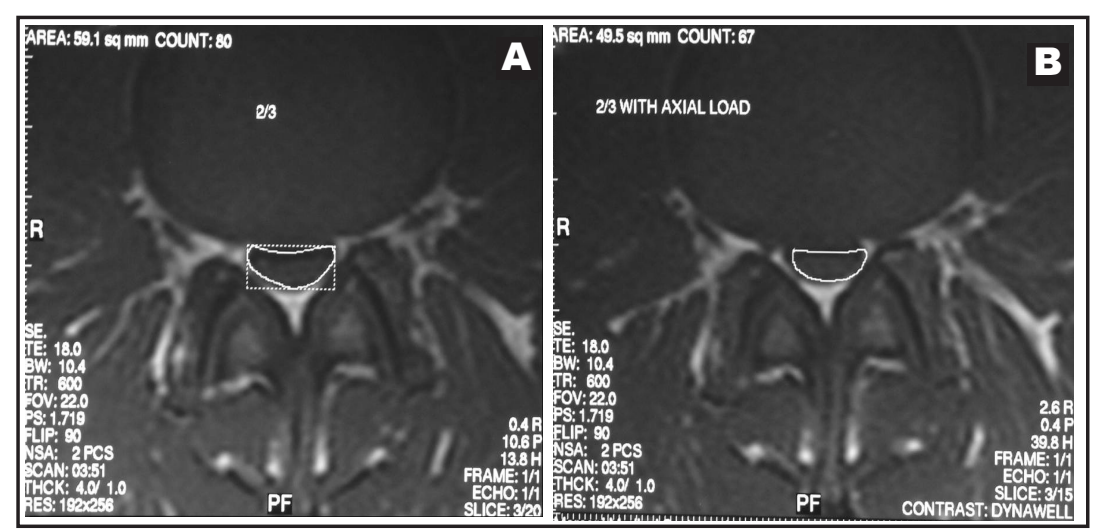

Fig 4. A comparison between central AP diameters of two patients at the midpedicle of L3 is depicted. (A) Within normal limits at 13mm. (B) Stenotic at $9 \mathrm{~mm}$ as the result of congenitally short pedicles

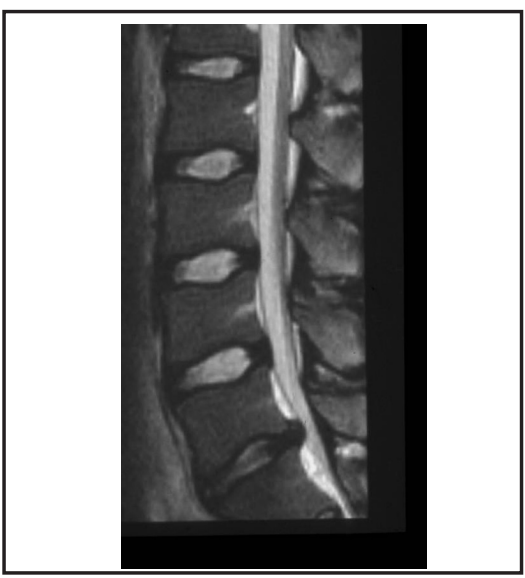

Fig 5. T2-weighted sagittal image of the lumbar spine reveals how even a mild disc prolapse can result in marked stenosis given a pre-existing congenital or developmental stenosis on the state of the herniated disc material (calcified in the latter). The condition of a "soft" stenosis will render it susceptible to forces imposed by dynamic mechanical alterations (Figs. 8-9) (3).

\section{Middle Column}

The middle column is also referred to as the neuroaxial canals, and is the actual site of the stenosis. Therefore, there is little in the way of contribution from the structures of the middle column contributing to the stenotic process. Pathological conditions which may arise and cause narrowing here include dural ectasia/ hyperplasia from inflammatory conditions (e.g. ankylosing spondylosis), lipomatosis, epidural abscess or hematoma, arteriovascular malformations, and neoplasm. All are uncommon; however, the astute clinician must always consider their possibility due to the morbidity and mortality associated with delayed diagnosis especially concerning neoplasm. Tumors are classified according to their location: 1) osseous, 2) extradural, 3) intradural/ extramedullary, or 4) intramedullary. Extradural tumors are by far the most common and fortunately almost always benign (if primary), but they still may cause significant mass affect and sequelae. Conversely, intramedullary tumors are the least common but usually malignant type of primary neurogenic tumor. Secondary tumors from metastatic disease are most commonly seen in the osseous elements and epidural space (Figs. 10-11)(8-10).

\section{Posterior Column}

The posterior elements may be implicated in stenosis secondary to congenital malformations of the neural arch, isthmic spondylolysis with spondylolisthesis, retrolisthesis, and lateral olisthesis (11, 12). Similar to the anterior column, stenosis most commonly results as a consequence of degeneration. Degenerative processes of the anterior and posterior columns often co-exist and may attenuate one another (13). For example, collapse of an intervertebral disc results in greater sagittal load sharing by the zygapophyseal joints. The resultant stress shielding can cause hypertrophic facet arthropathy with stenosis secondary to osseous encroachment. Hypertrophic facet arthropathy alters the normal morphology and orientation of the facet joints as well, compromising their biomechanical integrity. Facet subluxation ensues as a consequence of 


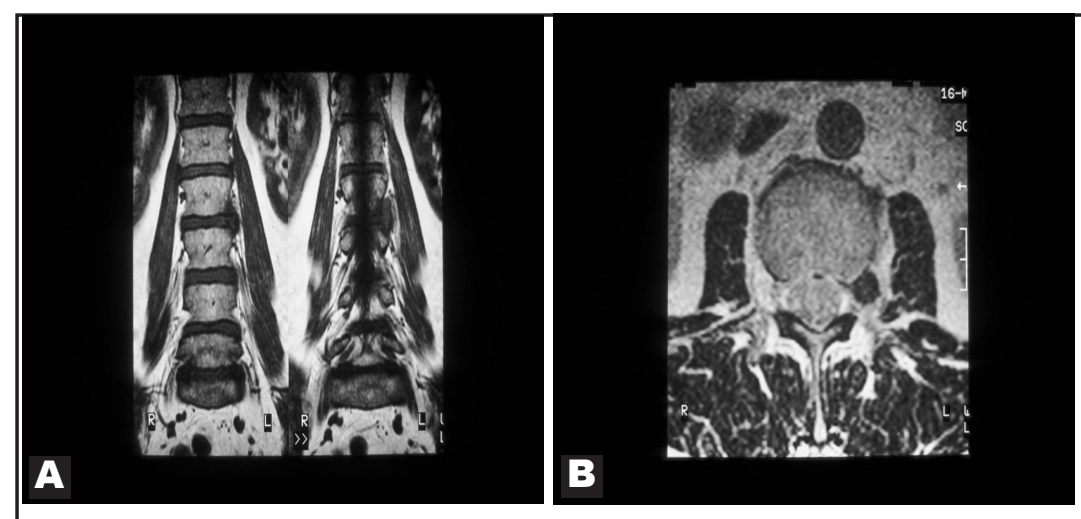

Fig 7. (A) Coronal lumbar MRI reveals a low signal mass lateral to the L2 and L3 pedicles (in the exit zone). (B) Transaxial T2-weighted MRI supports the finding of an old "far out" disc sequestrum

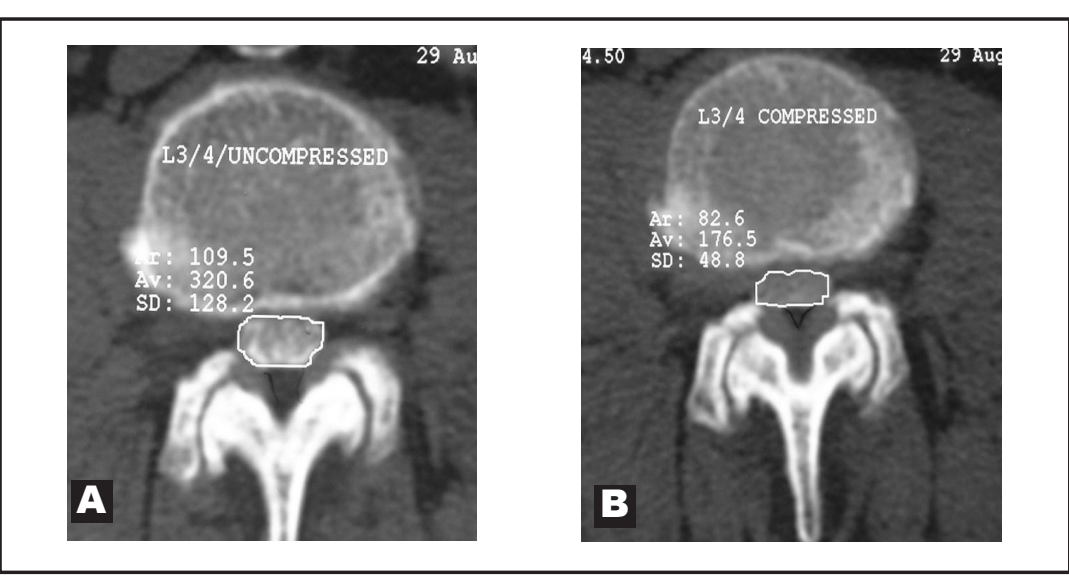

Fig 8. These images were acquired (A) before and (B) after application of the Dyanawell compression device during a lumbar post-myelography/ computerized tomography examination. There is a marked change in the central canal contour (white encircled area) upon loading the spine secondary to annular bulging and subluxation of the posterior joints
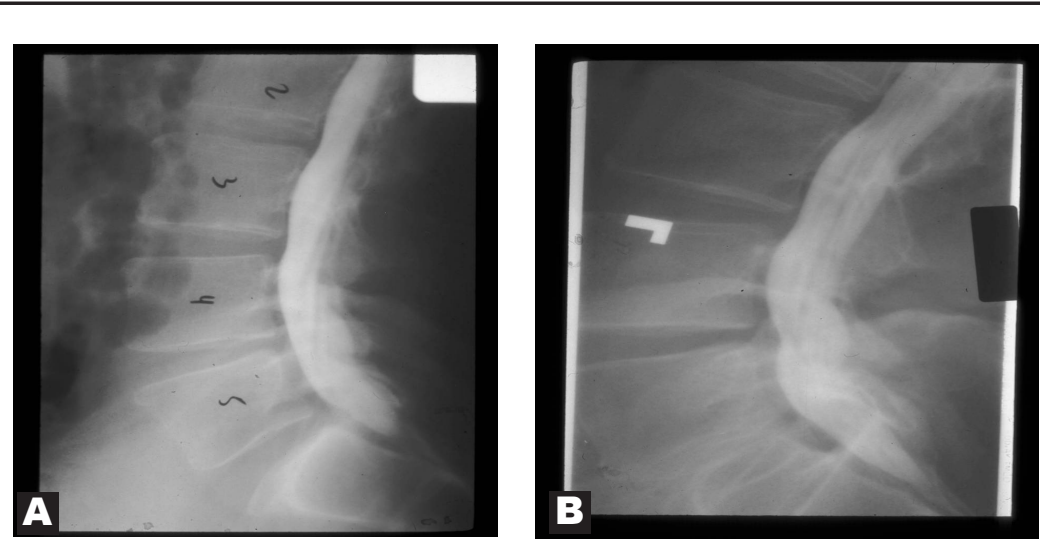

Fig 9. Standing sagittal lumbar myelography projections performed in $(A)$ neutral and (B) extension. Visualize the contrast column adjacent the L4-5 disc to appreciate the dynamic posterior prolapse upon extension dysfunction and greater load-sharing demands. Degenerative spondylolisthesis may result from this chronic process, contributing to further osseous stenosis (14, 15). For example, asymmetric degenerative collapse of the L5/S1 interspace with a spondylolisthesis may result in apposition of the L5 transverse process base to the adjacent sacral ala. The L5 anterior ramus is consequently trapped in its exit zone (so called "far-out" stenosis). Grade I degenerative spondylolisthesis is usually asymptomatic except in the context of combined stenosis or new pathological process (e.g., acute disc herniation, tumor) (1). In addition to causing osseous encroachment, degenerative spondylolisthesis generates a shear force upon the intervertebral disc and anterior or posterior longitudinal ligaments. Thus, the anterior column is subject to further degeneration in a vicious cycle. Other degenerative processes of the posterior elements implicated in stenosis include synovial cyst formation and the development of a redundant ligamentum flavum. A synovial cyst stemming from a diseased facet joint can certainly cause encroachment upon the canal(s) (Fig. 12). A redundant ligamentum flavum may buckle and bulge into the central canal (3).

\section{IMAGING MOdALITIES}

High resolution, multi-planar imaging is essential to appreciate the vertical, $\mathrm{AP}$, and transverse dimensions of the central and root canals. Modality selection must account for the strengths and limitations both CT and MRI have in depicting normal anatomy and pathology.

\section{Magnetic Resonance Imaging (MRI)}

MRI is the gold standard in evaluating soft tissue processes. It is unsurpassed in depicting intrathecal anatomy (morphology and position of the cord, conus, and nerve roots), neurogenic edema, hemorrhage, myelomalacia, syrinx, epidural hematoma or abscess, lipomatosis, synovial cyst, ligamentous and discogenic abnormalities, epidural or perineural fibrosis, and inflammatory, infiltrative, or neoplastic conditions (16-18). The addition of gadolinium enhancement will greatly increase its sensitivity for differentiation of a benign versus malignant syrinx, arteriovenous malformations, infarcts, myelomalacia, myositis, multiple sclerosis, sarcoidosis, and tumors-including leptomeningeal spread (8-10). Limitations of MRI include variable sig- 


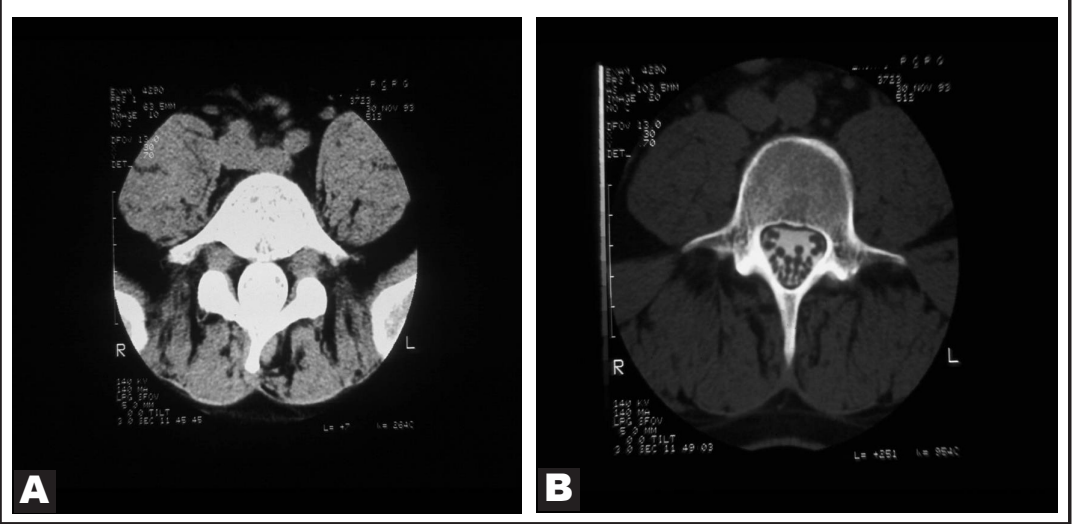

Fig 10. (A) Transaxial post-myelography lumbar CT in soft tissue window settings discloses huge L5 dorsal root ganglion. (B) The same patient with bone window settings at the L4 vertebral level demonstrates very conspicuous, rather large dural root sleeves. Findings such as these suggest a pathological primary neurogenic process resulting in hypertrophic nerves, such as Dejerine-Sottos or Charcot-Marie-Tooth
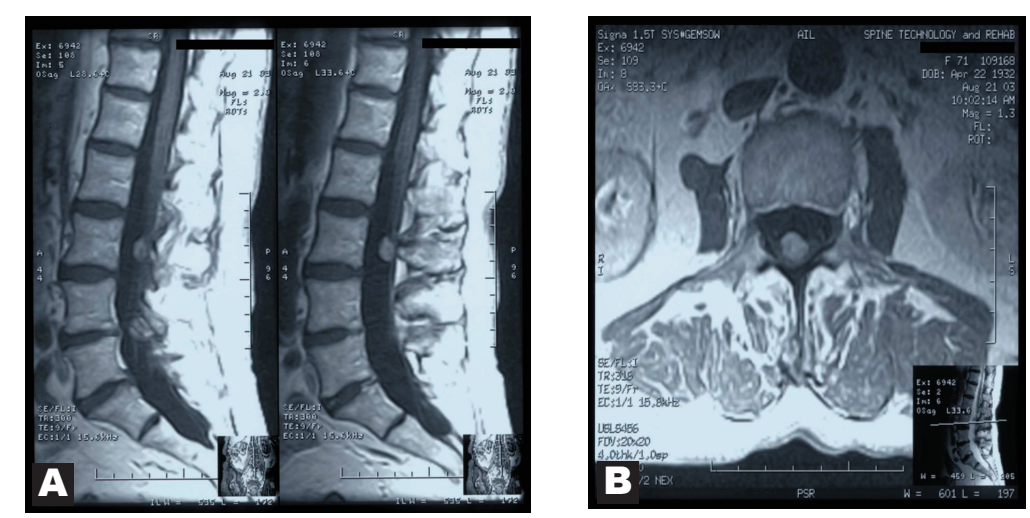

Fig 11. Enhancing neurofibroma of the filum terminale is demonstrated on T1-wieghted sagittal (A) and axial images (B). The lesion occupies roughly $40 \%$ of the volume of the central canal

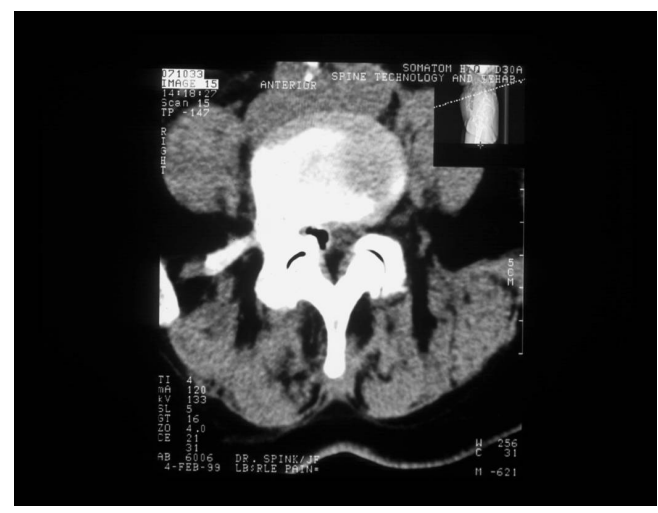

Fig 12. There is a low-density mass in the right L5 lateral recess (entry zone) and within the adjacent zygapophyseal joint, representing vacuum phenomenon within a synovial cyst and the joint nal intensity in degenerative osseous ridges (making it difficult to distinguish hard versus soft disc pathology), magnetic susceptibility, and thicker slices compared to CT. While gradient echo pulse sequences are beneficial in defining the etiology of stenosis, they are most prone to magnetic susceptibility artifact, which may exaggerate or obscure the extent of stenosis (16). Spondylolysis can be detected on MRI, but the sensitivity is less than CT especially in detecting associated fragments (19). MRI is contraindicated in patients with certain implantable ferromagnetic devices and relatively contraindicated in pregnancy (20).

\section{Computed Tomography (CT)}

Computed tomography (CT) provides superior resolution of the osseous dimensions of the canals and excellent delineation of the posterior elements. Degenerative, erosive, and destructive changes of the zygapophyseal joints as well as facet orientation, capsular calcification, and bony defects are all better visualized by CT. While MRI is more sensitive in detecting most disc abnormalities, CT is superior for differentiating hard versus soft disc pathology $(16,21-24)$. Contraindications include pregnancy and previously high total radiation exposures.

\section{Myelography}

Myelography historically has been considered the gold standard for evaluating the compression affect upon the thecal sac (25). It has since been replaced by MRI and is indicated only when MRI is not an option (i.e., contraindicated or quality jeopardized by metallic artifact status-post spinal fusion with instrumentation) $(20,26,27)$.

\section{Choosing the Best Imaging Modality}

As previously stated, the true osseous diameter of the central canal is best obtained from axial images orthogonal to the long axis of the canal. This should be measured from the anterior base of the spinous process to the midpoint of the posterior vertebral body. CT provides superior delineation of osseous structures compared to MRI and is therefore the modality of choice for this purpose (28). However, it should be noted that osseous stenosis does not necessarily correlate with the neurogenic symptoms of spinal stenosis. Bolender et al (25) found that the cross-sectional area of the thecal 


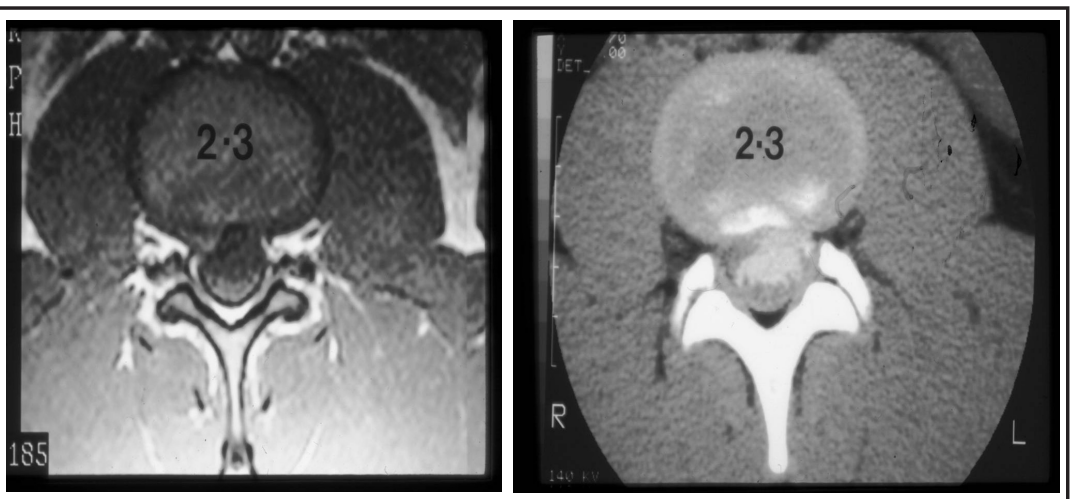

Fig 13. A mild right paracentral L2-3 prolapse is compared between (A) TIweighted axial image and (B) post-myelography CT image. Deformation of the thecal sac is appreciated rather equally

sac correlated best to stenotic symptoms. This is not entirely surprising since osseous stenosis, albeit the most common, is not the only contributor as discussed. The influence of ligamentous, discogenic, and other implicated structures must be taken into account. Additionally, the best predictor of the clinical course in myelopathic patients including surgical interventional outcome is the degree of cord compression (reduced thecal sac and cord volumes) and extent of associated pathological changes (i.e., myelomalacia) $(29,30)$. Clearly, MRI with its excellent depiction of soft tissue is best suited for measuring these volumes and demonstrating pathological changes. The limitation in this instance is that cross-sectional area is not easily measured unless done at the time of the study, which is not routinely performed.

Comparing surgical findings with those of MRI, CT, and myelography, Modic et al $(20,28)$ determined MRI findings congruent in $77 \%$, CT findings in $79 \%$, and myelographic findings in $54 \%$. The limitations of this study were that it did not include multi-planar reformations for CT (potentially increasing the sensitivity) and no differentiation was made between central and root canal stenosis. Nonetheless, MRI and CT appear to be relatively equal in their ability to identify stenosis on the whole (Fig. 13) $(23,27)$. When deciding on the optimal imaging modality, the ordering physician must use his or her own clinical judgment in predicting what structures may be implicated. For example, CT may be the better choice if arthritis is known or suspected, while MRI is warranted if the onset of symptoms is abrupt or neurological deficits are present. Some authors comment that MRI and CT are complimentary and that both may be necessary $(16,27,31)$.

\section{Conclusion}

Modern multi-planar imaging studies provide excellent visualization of the osteoligamentous conduits and neurovascular contents of the spine. These examinations should always be systematically selected and applied according to a thorough history and physical examination. The biomechanics of the injury and the pathophysiology of the disease process in question should always be considered. Understanding the unique properties of each diagnostic modality (as well as the combined benefits of various modalities) will enhance the clinician's diagnostic yield and ensure cost effectiveness. MRI provides the best visualization of soft tissue structures, while CT is the diagnosous structures. The strengths and limitations of spine imaging modalities should be factored into the selection process. It cannot be over-emphasized that abnormalities on imaging may not be responsible for the symptoms being investigated $(7,32)$. Presumptive correlations render "false-positives" that may lead to misguided treatment efforts. Clinical examination and physiological studies, such as image-guided injections and electrodiagnostics, should be implemented to confirm or refute the contribution and significance of structural pathology. tic modality of choice for evaluating osse-
Author Affiliation:

Joseph D. Fortin, DO

Medical Director

Spine Technology and Rehabilitation, $\mathrm{PC}$

Clinical Professor

Indiana University School Medicine Jefferson Center, 7230 Engle Road

Suite 210

Fort Wayne, IN 46804

E-mail: fortin@pol.net

\section{Michael T. Wheeler, DO}

Fellow

Spine Technology And Rehabilitation, $\mathrm{PC}$

Jefferson Center, 7230 Engle Road

Suite 210

Fort Wayne, IN 46804

\section{References}

1. Verbiest H. Fallacies of the present definition, nomenclature, and classification of the stenosis of the lumbar vertebral canal. Spine 1976; 1:217-225.

2. Arnoldi CC, Brodsky AE, Cauchoix J et al. Lumbar spinal stenosis and nerve root entrapment syndromes: Definition and classification. Clin Orthop Rel Res 1976; 115:4-5.

3. Rauschning W. Normal and pathologic anatomy of the lumbar root canals. Spine 1987; 12:1008-1019.

4. Wiltse L. Far-out syndrome. In Rothman SLG, Glenn WV Jr (eds): Multiplanar CT of the Spine. University Park Press, Baltimore, 1985.

5. Edwards WC, LaRocca H. The developmental segmental sagittal diameter of the cervical spinal canal in patients with cervical spondylosis. Spine 1983; 8:20-27.

6. Bohatirchuk F. The ageing vertebral column (macro- and historadiographical study). BrJ Radiol 1955; 28:389-404.

7. Wiesel SW, Tsourmas N, Feffer HL et al. A study of computer-assisted tomography. I. The incidence of positive CAT scans in an asymptomatic group of patients. Spine 1984; 9:549-556.

8. Sze G, Bravo S, Krol G. Spinal lesions. Quantitative and qualitative temporal evolution of gadopentetate dimeglumine enhancement in MR imaging. Radiology 1989; 170:849-856.

9. Sze G, Krol G, Zimmerman RD et al. Intramedullary disease of the spine: Diagnosis using gadolinium-DTPA-enhanced MR imaging. Am J Roentgenol 1988; 151:1193-1204.

10. Sze G, Krol G, Zimmerman RD et al. Malignant extradural spinal tumors: MR imaging with gadolinium-DTPA. Radiology 1988; 167:217-223.

11. Edelson JG, Nathan H. Nerve root compression in spondylolysis and spondylolis- 
thesis. J Bone Joint Surg 1986; 68B:596599.

12. Rothman SL, Glenn WV Jr. CT multiplanar reconstruction in 253 cases of lumbar spondylolysis. Am J Neuroradiol 1984; 5: 81-90.

13. Szypryt EP, Twining P, Mulholland RC et al The prevalence of disc degeneration associated with neural arch defects of the lumbar spine assessed by magnetic resonance imaging. Spine 1989; 14:977-981.

14. MacNab I. Spondylolisthesis with an intact neural arch, the so-called pseudo-spondylolistheses. J Bone Joint Surg 1950; 32B: 325-333.

15. Sato K, Wakamatsu E, Yoshizumi A et al. The configuration of the laminas and facet joints in degenerative spondylolisthesis: A clinicoradiologic study. Spine 1989; 14: 1265-1271.

16. Maravilla KR, Hartling RP. Imaging decisions in degenerative spinal disease: $A$ practical approach. MRI Decisions 1988; 2:2-13.

17. VanDyke C, Ross JS, Tkach J et al. Gradient-echo MR imaging of the cervical spine: Evaluation of extradural disease. Am Roentgenol 1989; 153:393-398.

18. Williams AL, Haughton VM, Pojunas KW et al. Differentiation of intramedullary neoplasms and cysts by MR. Am J Roentgenol 1987; 149:159-164.
19. Grenier N, Kressel HY, Schiebler ML et al. Isthmic spondylolysis of the lumbar spine: MR imaging at 1.5T. Radiology 1989; 170: 489-493.

20. Shellock FG: MR imaging of the metallic implants and materials: A compilation of the literature. Am J Roentgenol 1988; 151: 811-814.

21. Lancourt JE, Glenn WV Jr, Wiltse LL. Multiplanar computerized tomography in the normal spine and in the diagnosis of spinal stenosis: A gross anatomic-computerized tomographic correlation. Spine 1979; 4:379-390.

22. McAfee PC, Ullrich CG, Yuan HA et al. Computed tomography in degenerative spinal stenosis. Clin Orthop Rel Res 1981; 161: 221-234.

23. Schnebel B, Kingston $S$, Watkins $R$ et al. Comparison of MRI to contrast CT in the diagnosis of spinal stenosis. Spine 1989; 14:332-337.

24. Gunzburg R, Sandhu A, Fraser RD. The value of computerized tomography in determining lumbar facet joint orientation. J Spinal Dis 1989; 2:170-175.

25. Bolender NF, Schonstrom NSR, Spengler DM. Role of computed tomography and myelography in the diagnosis of central spinal stenosis. J Bone Joint Surg 1985; 67A:240-245.

26. Brown BM, Schwartz RH, Frank $E$ et al. Preoperative evaluation of cervical radiculopathy and myelopathy by surfacecoil MR imaging. Am J Roentgenol 1988; 151:1205-1212.

27. Modic MT, Masaryk T, Boumphrey F et al. Lumbar herniated disc disease and canal stenosis: Prospective evaluation by surface coil MR, CT and myelography. Am J Roentgenol 1986; 147:757-765.

28. Stanley JH, Schabel SI, Frey GD et al. Quantitative analysis of the cervical spinal canal by computed tomography. Neuroradiology 1986; 28:139-143.

29. Fujiwara K, Yonenobu K, Ebara S et al. The prognosis of surgery for cervical compression myelopathy: An analysis of the factors involved. J Bone Joint Surg 1989; 71B: 393-398.

30. Fujiwara K, Yonenobu K,Hiroshima K et al. Morphometry of the cervical spinal cord and its relation to pathology in cases with compression myelopathy. Spine 1988; 13 : 1212-1216.

31. Modic MT, Masaryk TJ, Mulopulos GP et al. Cervical radiculopathy: Prospective evaluation with surface coil MR imaging, CT with metrizamide, and metrizamide myelography. Radiology 1986; 161:753-759.

32. Teresi LM, Lufkin RB, Reicher MA et al. Asymptomatic degenerative disc disease and spondylosis of the cervical spine: MR imaging. Radiology 1987; 164:83-88. 
